\title{
Role of clinical neurophysiological tests in evaluation of erectile dysfunction in people with spinal cord disorders
}

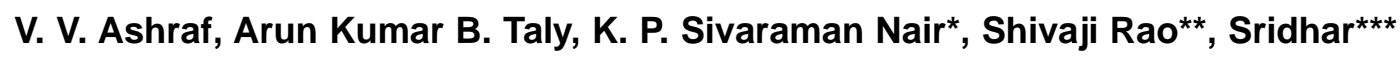 \\ Department of Neurology, Malabar Institute of Medical Sciences, Calicut - 673016, Departments of ${ }^{*}$ sychiatric and Neurological Rehabilita- \\ tion, ${ }^{* *}$ Biostatistics, ${ }^{* * *}$ Clinical Neurophysiology, National Institute of Mental Health and Neurosciences, Bangalore - 560 029, India
}

\begin{abstract}
Background: While erectile dysfunction is frequent among people with disorders of the spinal cord, the role of various clinical neurophysiological tests in assessment is not clear. Aims: To study the role of clinical neurophysiological investigations in assessing erectile dysfunction among men with spinal cord disorders. Setting: National Institute of Mental Health and Neurosciences, India. Design: Survey. Materials and Methods: Subjects with a score of 21 or less on the International Index of Erectile Function-5 were classified as with erectile dysfunction and with a score of more than 21 as without erectile dysfunction. Clinical neurophysiological studies done were Sympathetic Skin Response from limbs, posterior tibial sensory evoked potential, pudendal sensory potential and bulbocavernous reflex. Statistical Analyses: Chi-square test. Results: Among 40 subjects 26 had erectile dysfunction. The frequency of abnormalities in clinical neurophysiological studies were: pudendal sensory evoked potentials - 16, posterior tibial sensory evoked potentials - 26 , bulbocavernous reflex - 5 , sympathetic skin response from sole - 24 and, sympathetic skin response from palm - 18. Significant associations were noted between erectile dysfunction and abnormal pudendal sensory evoked potentials $(P=0.0479)$, and absent sympathetic skin response from palm $(P=0.0279)$ and sole $(P<0.001)$. There was no correlation between erectile dysfunction and posterior tibial sensory evoked potentials $(P=0.133)$ or bulbocavernous reflex $(P=0.418)$. Sympathetic skin response from sole was most sensitive (80.8\%) and had best positive (87.5\%) and negative predictive $(68.8 \%)$ values. The specificity of these three tests was $78.6 \%$. Conclusions: Sympathetic skin response from the sole of the foot was the most sensitive and specific clinical neurophysiological test for erectile dysfunction in spinal cord disorders.
\end{abstract}

Key Words: Bulbocavernous reflex, erectile dysfunction, sensory evoked responses, spinal cord injury, sympathetic skin response

\section{Introduction}

Prevalence of erectile dysfunction (ED) among men with spinal cord disorders (SCD) varies from $40 \%$ to $60 \% .^{[1,2]}$ Different clinical neurophysiological investigations have been used in the evaluation of ED ${ }^{[3]}$ Pudendal sensory evoked potentials (SEP) recorded with scalp electrodes provides objective means of testing the afferent pathways from the dorsal nerve of the penis to the sensory cortex. The recording of bulbocavernosus reflex (BCR) tests the integrity of sacral roots and conus medullaris. ${ }^{[2]}$ Sympathetic skin response (SSR) from the sole and genitalia is a valuable investigation in the evaluation of ED in patients with a variety of neurological disorders including SCD. ${ }^{[4]}$ Afferent pathways of SSR can be evaluated by recording SSR from the palm on stimulating the posterior tibila nerve (PTN) below the level of the lesion. ${ }^{[5]}$ However, there are no reports on the efficacy of this technique in evaluating ED. The purpose of this study was to evaluate the sensitivity, specificity, positive and negative predictive values of PSEP, posterior tibial SEP, SSR, BCR in the evaluation of ED in subjects with SCD.

\section{Materials and Methods}

This cross-sectional survey was carried out from February 2001 to December 2001 and involved 40 men between the age group of 1850 years with SCD. Patients with features of other diseases that can cause ED including peripheral vascular disease, diabetes and those with associated peripheral neuropathy or postganglionic lesion as evident by absent sural sensory nerve action potential were excluded from the study. Patients with lesions involving the conus medullaris or cauda equina were also excluded from the study. All patients were clinically evaluated as per the guidelines in the International booklet for standard neurological and functional classification of spinal cord injury. ${ }^{[6]}$ For the purpose of this study, the highest abnormal spinal cord segment on MRI was considered as the level of lesion. Erectile Dysfunction was assessed using the 5-item version of the 'Interna- 
tional index of erectile function (IIEF-5). ${ }^{[7]}$ Subjects who had a score of 21 or less were classified as with ED and others as without ED. This cut-off score had a sensitivity of $98 \%$ and specificity of $88 \%$ in the diagnosis of ED. ${ }^{[7]}$

Clinical neurophysiological studies carried out included: conduction studies of common peroneal, posterior tibial and sural nerves, SSR, PSEP, PTSEPs and BCR. Motor and sensory nerve conduction studies were done in all patients on Nihon-Kohden Neuropack sigma apparatus (Japan) at room temperature using the technique described earlier. Motor conduction study of sural, posterior tibial and common peroneal nerves was done. Sensory conduction studies were carried out to rule out associated sensory neuropathy. Subjects with abnormal motor or sensory conduction studies were excluded from the study.

Efferent pathway of SSR was evaluated by recording from the sole after stimulating the median nerve at the wrist. ${ }^{[8-10]}$ Afferent pathways were evaluated by recording from the palm after stimulating the posterior tibial nerve at the ankle (below the level of lesion). ${ }^{[5]}$ Standard surface disc electrodes were placed on the palm and dorsum of the hand and the sole and dorsum of the right foot. Stimuli consisted of square wave electric pulses of 0.2 msec duration and 20$40 \mathrm{~mA}$. An interval of $30 \mathrm{sec}$ was maintained between individual stimuli to avoid habituation. Sweep speed of $500 \mathrm{msc} / \mathrm{cm}$ and gains of $200-500 \mathrm{mV} / \mathrm{cm}$ were used with low and high filter setting of $2 \mathrm{~Hz}$ and $2 \mathrm{KHz}$ respectively. A minimum of ten random stimuli were given at each site before considering the SSR to be absent. Only absence of SSR was considered as abnormal. ${ }^{[9,10]}$

Posterior tibial SEP were recorded by stimulating the posterior tibial nerves in all patients. Active recording electrode was placed at $\mathrm{CZ}, 2 \mathrm{~cm}$ behind the central area $(\mathrm{CZ})$ and reference at frontal area (FZ) according to the international ten-twenty electrode system. Stimuli consisted of square pulse electric stimuli of 0.2 msec duration. Current strengths that were just sufficient to produce a visible twitch in the plantar flexors of toes in the foot were used. Rate of stimulation was $2 /$ sec. A minimum of 1000 responses was averaged. ${ }^{[11]}$ Latency was measured from the stimulus artifact to the first positive component, which corresponded to $\mathrm{P}_{37}$. This latency was compared with control values established for the laboratory. Value was considered abnormal if it was more than 45.5 msec (3 S.D. above mean) or if there was no waveform.

Pudendal SEP recording from the scalp was done by stimulating the base of the penis using ring electrodes. Square wave single pulses of 0.1 msec duration at a frequency of 2 stimulations per second were administered. The sensory threshold was defined as the lowest current perception. Stimulus intensity of three times the threshold was used to obtain cortical evoked potentials from the midline of the scalp $2 \mathrm{~cm}$ behind the central vertex $\mathrm{Cz}$. Reference electrode was placed at $\mathrm{Fz}$ and a minimum of 1000 responses were averaged.$^{[4,11,12]}$ To ensure consistency of the response, the recording was done twice. The latency was measured from the stimulus artifact to the first positive peak or P1 $\left(\mathrm{P}_{37}\right)$. Value was considered abnormal if this latency was more than 47.3 msec (i.e. 2 standard deviations more than the normal control values) or if there was no waveform.

BCR was elicited by stimulating the pudendal nerve at the shaft of the penis by two surface ring electrodes. Response was recorded by means of bipolar surface electrodes midway between the scrotum and anus. Reference electrode was placed on the medial aspect of the thigh close to the scrotum. Sweep speed was set to $8 \mathrm{msec} / \mathrm{div}$ and filter band pass to $10 \mathrm{~Hz}-3 \mathrm{KHz}$. Forty sweeps were averaged. Latency was determined from stimulus artifact to first negative deflection of the response. It was considered to be abnormal when absent or when the onset latency was more than 44.4 msec. ${ }^{[12]}$ The study had clearance of the ethical committee of the institute and all subjects were enrolled after obtaining informed consent.

\section{Statistical analysis}

Significance of abnormal clinical neurophysiological tests was assessed with chi-square test. $P$ value of less than 0.05 was considered significant. The sensitivity of a test is defined as the percentage of persons with ED who had abnormal test results. Specificity is the percentage of persons without ED who had normal test results. Positive predictive value is the percentage of persons with abnormal results who actually had ED. Negative predictive value is probability of ED being absent if the test is normal. Sensitivity, specificity, positive and negative predictive values of all the clinical neurphysiological tests significantly associated with ED are calculated.

\section{Results}

The mean age of these patients was $32.8 \pm 8.9$ years (range: $18-48$ yrs). The duration of the neurological symptoms varied from 30 days to 6840 days; $($ mean $=584.4+1129.3$ days, median 150 days). The etiologies of SCD were: acute transverse myelitis-18, trauma-1, tumor-6, disc prolapse-3, tuberculosis of spine-3, archnoiditis-2, tropical spastic paraplegia2 , tuberculosis with epidural abscess-1, flurosis with ossified posterior longitudinal ligament causing cord compression-1, atlantoaxial dislocation-1, and cause not known-2. Ten subjects had tetraplegia and 30 paraplegia. Six subjects (15\%) had complete cord lesion (Grade A) and 34(80\%) patients had incomplete cord lesion [Grade B-6; Grade C-10; Grade D-17; Grade E-1]. All the forty patients underwent MRI and the highest vertebral level was in the cervical cord in 17 patients, above the sixth thoracic segment in 20 patients and below the sixth thoracic segment level in 3 patients $\left(8^{\text {th }}\right.$ thoracic segment-1, $10^{\text {th }}$ thoracic segment- 1 and $12^{\text {th }}$ thoracic segment-1). Twenty-six patients (65\%) had ED. SSR was absent from the sole on median nerve stimulation in 24 subjects and from the palm on stimulation of the posterior tibial nerve in 15. Pudendal SEP was abnormal in 17 subjects; prolonged latency was seen in 8 and absent response in 9 patients. Posterior tibial SEP was abnormal in 26 subjects. BCR was absent in 2 and in another 3 patients latency was prolonged.

Absence of SSR from the palm and sole and abnormal $\mathrm{Pu}$ dendal SEP were significantly associated with ED (Table 1). There was no association between abnormal posterior tibial SEP and BCR with ED (Table 1). SSR in the lower limbs to median nerve stimulation was the most sensitive of all the clinical neurophysiological investigations. SSR from the palm, SSR from the sole and pudendal SEP all had the same specificity (Table 2 ). 


\begin{tabular}{|c|c|c|c|}
\hline & $\begin{array}{l}\text { With ED* } \\
(n=26)\end{array}$ & $\begin{array}{l}\text { Without ED* } \\
\quad(n=14)\end{array}$ & $P$ value \\
\hline Absent $\mathrm{SSR}^{\dagger}$ from sole of foot & 21 & 3 & $0 .<001^{\ddagger}$ \\
\hline $\begin{array}{l}\text { Absent } \mathrm{SSR}^{+} \text {in palm onPT } \\
\text { sstimulation }\end{array}$ & 15 & 3 & $0.0279^{\ddagger}$ \\
\hline Abnormal PT§ SEPQ" & 19 & 7 & $0.1334^{\ddagger}$ \\
\hline Abnormal Pudendal SEPQ" & 14 & 3 & $0.0479^{\ddagger}$ \\
\hline Abnormal Bulbocavernous reflex & 4 & 1 & $0.418^{\ddagger}$ \\
\hline
\end{tabular}

${ }^{\star}$ ED - Erectile dysfunction, ${ }^{+S S R}$ - Sympathetic skin response, ${ }^{\ddagger}$ - Chi-square test, §-PT-Posterior tibial nerve, ISEP - Sensory evoked potential

\begin{tabular}{|c|c|c|c|c|}
\hline Test & $\begin{array}{c}\text { Sensitivity } \\
(\%)\end{array}$ & $\begin{array}{c}\text { Specificity } \\
(\%)\end{array}$ & $\begin{array}{l}\text { Positive } \\
\text { predictive } \\
\text { value (\%) }\end{array}$ & $\begin{array}{l}\text { Negative } \\
\text { predictive } \\
\text { value (\%) }\end{array}$ \\
\hline SSR $^{*}$ from Sole & 80.8 & 78.6 & 87.5 & 68.8 \\
\hline SSR $^{*}$ from Palm & 57.7 & 78.6 & 83.3 & 50.0 \\
\hline Pudendal SEP ${ }^{\dagger}$ & 53.8 & 78.6 & 82.4 & 47.8 \\
\hline
\end{tabular}

*SSR-Sympathetic skin response, ₹SEP- Sensory evoked potential

\section{Discussion}

Sexual dysfunction is frequent among men with SCD. Erection is often preserved among subjects with incomplete lesions of spinal cord above sacral segments. Bors and Comarr reported ED in $78 \%$ of patients with SCI. ${ }^{[13]}$ We noted ED in only $65 \%$ of subjects. We excluded subjects with cauda equina lesions. This may be the reason for the difference in the frequency of ED with other series.

Sympathetic skin response is a polysynaptic reflex generated possibly in the posterior hypothalamus. The afferent limb involves neurons arising from the intermediolateral column of the spinal cord. ${ }^{[14]}$ Ertekin et al found SSR to be useful in evaluating ED due to peripheral neuropathies. ${ }^{[15]}$ Kunesh et al reported that among the battery of tests applied to men with ED, lower limb SSR was most frequently abnormal. ${ }^{[16]}$ Most studies on SSR in SCD focused on its relationship with the clinical level, autonomic dysreflexia and bladder functions. ${ }^{[14,17]}$ Courtois et al found that subjects with SCI who have a normal SSR maintain psychogenic erections. ${ }^{[4]}$ In the present study absent SSR from the sole was the most sensitive test for ED (Table 2). The SSR is the activity of sudomotor glands stimulated by the sympathetic nervous system. Erection occurs due to the relaxation of the arterial smooth muscles through the parasympathetic nervous system. The sympathetic neurons do not have a direct role. The good correlation between $\mathrm{ED}$ and absent SSR in the sole of the foot may be due to the proximity of these tracts within the spinal cord.

SSR from the palm to PTN stimulation at the ankle assesses the afferent pathways of SSR in the spinal cord. ${ }^{[5]}$ In the present study, absent SSR in the palm to PTN stimulation was significantly associated with ED. However, this test was less sensitive than SSR from the sole and had the same specificity. This is the first study that evaluated this technique in $\mathrm{ED}$.

In the present study abnormal BCR is not associated with ED (Table 1). None of the subjects in this study had lesions involving the sacral segments or cauda equine. BCR is mediated by large diameter motor efferents while erection is mediated by small diameter autonomic fibers. These may be the reasons for poor correlation of BCR with ED in this study. Abnormal BCR in 5 subjects may indicate involvement of conus medullaris not detected by other tests.

Pudendal SEP provides information about afferent pathways from the dorsal nerve of the penis to the sensory motor cortex. The electrical impulses stimulated large diameter somatic sensory fibers. Deldovici and Fowler demonstrated abnormalities of pudendal SEP in $33 \%$ to $55 \%$ of the subjects with SCD and ED. ${ }^{[18]}$ We also noted that abnormal pudendal SEP is significantly associated with ED (Table 1). Ghezzi observed that in 6 patients with MS without ED, pudendal SEP was abnormal in 5 patients indicating its poor specificity. ${ }^{[11]}$ In contrast, in the present study specificity of this test was $78 \%$. This may be due to difference in the etiologies of SCD.

Pudendal SEP is more cumbersome and unpleasant, compared to posterior tibial SEP. Rodi et al reported that in subjects with MS and bladder symptoms, posterior tibial SEPs were abnormal in $90 \%$ whereas pudendal SEP were abnormal in only 40\% ${ }^{[19]}$ Deldovici and Fowler also reported similar disparity between pudendal and posterior tibial SEPs. ${ }^{[18]}$ In the present study also posterior tibial SEP was more frequently abnormal compared to pudendal SEP (Table 1). However, there was no significant association between ED and abnormal posterior tibial SEP (Table 1). While posterior tibial SEP has a unilateral pathway in the spinal cord, pudendal SEP has bilateral pathways. This could be the reason for the disparity between these two responses. Pudendal SEP evaluates somatosensory pathways from the penis and should not be substituted with posterior tibial SEP while testing for ED.

In this study, clinical neurophysiological tests significantly associated with ED were pudendal SEP and SSR from the sole and palm. Among these tests SSR from the sole was the most sensitive. All tests had equal specificity (Table 2). Courtois et al and Schmidt et al also noted that SSR is a good test for ED in SCI. ${ }^{[4,20]}$ The erection is a complex process requiring coordinated functioning of the somatosensory, sympathetic and parasympathetic systems. The ED in SCD could be due to involvement of any or all of these. Hence a single test is not sufficient to evaluate ED in SCD. Pudendal SEP is a test of somatic sensory pathways and SSR of sympathetic activity. We recommend a combination of pudendal SEP and SSR for evaluation of ED in SCD. BCR may be required if the conus medullaris or cauda equina are involved. 


\section{Conclusions}

ED is common in men with SCD. Abnormal results of pudendal SEP and SSR from the palm and sole are significantly associated with ED. SSR from the sole is the most sensitive and specific clinical neurophysiological test of ED in subjects with SCD.

\section{Acknowledgement}

We thank Ms. Manjula Sharma for secretarial assistance.

\section{References}

1. Biering-Sorensen F, Sonksen J. Sexual functions spinal cord lesioned men. Spinal Cord 2001;39:455-70.

2. Ghezzi A, Malvestiti G, Baldini S, Zaffaroni M, Zibetti A. Erectile impotence in multiple sclerosis: A neurophysiological study. J Neurol 1995;242:123-6.

3. Fowler C.J. The neurology of male sexual dysfunction and its investigation by clinical neurophysiological methods. Br J Urol 1998;81:785-95.

4. Courtois F.J, Gonnaud PM, Charvier KF, Leriche A. Sympathetic skin responses and psychogenic erections in spinal cord injured men. Spinal Cord 1998;36:125-31.

5. Nair KPS, Taly AB, Rao S, Murali T. Afferent pathways of Svmpathetic skin response in spinal cord: A clinical and clinical neurophysiological study. J Neurol Sci 2001;187:77-80.

6. Ditumno .Jr .JF, Young W, Donovan WH, Creasy G. The international standards booklet for neurological and functional classification of spinal cord injury. Paraplegia 1994;32:70-80.

7. Rosen RC, Cappelleri JC, Smith MD, Lipsky J, Pena BM. Development and evaluation of an abridged, 5 -item version of the international index of erectile function (IIEF-5) as a diagnostic tool for erectile dysfunction. Int J Impot Res 1999;11:319-26.

8. Shahani BT, Halperin JJ, Boulu P, Cohen J. Sympathetic skin response- a method of assessing unmyelinated axon dysfunction in peripheral neuropathies. J Neurol Neurosurg Psychiatry 1984;47:536-42.

9. Arunodhaya GR, Taly AB. Sympathetic skin response: A decade later. J Neurol Sci 1995;129:81-9.

10. Shahani BT, Day T.J, Crose D, Khaleel N, Kneebone CS. R-R interval variation and sympathetic skin response in assessment of autonomic function in peripheral neuropathies. Arch Neurol 1990;47:659-94.

11. Ghezzi A, Callea L, Zaffaroni M, Montanini R, Tessera G. Motor potentials of bulbocavernosus muscle after transcranial and lumbar magnetic stimulation: Comparative study with bulbocavernous reflex and pudendal evoked potentials. J Neurol Neurosurg Psychiatry 1991;54:524-6.

12. Vodusek DM, Janko M. The bulbocavernous reflex. Brain 1990;113:813-20.

13. Bors EH, Comarr AE. Neurological disturbances of sexual function with special reference to 529 patients with spinal cord injury. Urol Surv 1960;10:191-222.

14. Nair KPS, Taly AB, Arunodhaya GR, Rao S, Murali T. Sympathetic skin response in myelopathies. Clin Auton Res 1998;8:207-11.

15. Ertekin C, Ertekin N, Mutlu S, Almis A, Akcam A. Skin potentials (SP) recorded from the extremities and genital regions in normal and impotent subjects. Acta Neurol Scand 1987;76:28-36

16. Kunesch E, Reiners K, Muller-Matheis V, Strohmeyer T, Ackermann R, Freund H. Neurological risk profile in organic erectile impotence. J Neurol Neurosurg Psychiatry 1992;55:275-81.

17. Curt A, Weinhardt C, Dietz V. Significance of sympathetic skin response in assessment of autonomic failure in patients with spinal cord injury. J Auton Nerv Syst 1996;61:175-80.

18. Delodovici ML, Fowler C.J. Clinical value of the pudendal somatosensory evoked potential. Electroencephalogr Clin Neurophysiol 1995;96:509-15.

19. Rodi Z, Vodusek D, Denislic M. Clinical uro-neurophysiological investigation in multiple sclerosis. Eur Neurol 1996;3:574-80.

20. Schmidt DM, Curt A, Hauri D, Schurch B. Clinical value of combined clinical neurophysiological and urodynamic recordings to assess sexual disorders in spinal cord injured men. Neurourol Urodyn 2003;22:314-21.

Accepted on 12.01.2005.

\section{Invited Comments}

Erectile dysfunction (ED) is a common neurological symptom, and patients with complete spinal cord injury almost inevitably face this problem. Brain and peripheral nerve diseases may cause ED as well. However, in various autonomic nervous system disorders, ED seems to be one of the most difficult areas to approach. This is because erection and related sexual matters are more difficult to reproduce in the laboratory for both technical and psycho-sociological reasons, than urinary and defecatory behaviors. Proper afferent signals for erection include not only somatosensory (reflexive erection), but also audiovisual, odor, and even imaginary stimulation (psychogenic erection). During sleep, REM stage-related erection also occurs (nocturnal penile tumescence). Erection depends on small-diameter (Adelta/ C) cholinergic (parasympathetic) and NOergic (non-adrenergic and non-cholinergic, NANC) fibers from the sacral cord that dilate the cavernous helical artery. We can reproduce erection pharmacologically (oral sildenafil or intracavernous papaverine injection), but not easily by electrical or magnetic stimulation. In the spinal cord injured pa- tients, these three types of erections are lost, because of disruption in the pathways that connect the hypothalamus and the sacral spinal cord, although reflexive erection can be spared.

Here, Ashraf et al described the results of five neurophysiological tests: palm sympathetic skin response (SSR) after posterior tibial nerve stimulation, sole SSR after median nerve stimulation, posterior tibial somatosensory evoked potential (SEP), pudendal SEP and bulbocavernosus reflex (BCR) in 26 spinal cord lesioned patients with ED and 14 patients without it. The authors found a relationship between ED and sole SSR, palm SSR, and pudendal SEP with sole SSR being the most sensitive. In spinal cord injured patients, sweating (sympathetic, cholinergic) and erection are not likely to correlate with each other at the peripheral level. However, the authors' results seem to indicate that sweating and erection pathways are located nearby within the spinal cord, therefore, they are likely to be damaged together. BCR efferent is a large-diameter somatic motor fiber to the striated sphincter muscle. This presumably explains why BCR and ED did not clearly corre- 
late with each other in the previous studies. Similarly, the afferent limb of pudendal SEP, as stimulated electrically (not painful), is a large-diameter somatic sensory fiber. This presumably explains why the sensitivity of pudendal SEP was less than that of sole SSR in the present study. The pathophysiology of ED per se is still not clear. However, the au- thors' results clearly indicated that sole SSR is useful as an object measure for ED in paraplegic patients.

Ryuji Sakakibara

Neurology, Chiba University, 1-8-1 Inohana Chuo-ku, Chiba 2608670, Japan.E-mail: sakakibara@faculty.chiba-u.jp 